\title{
Polarization ray tracing and polarization aberration compensation in reflective astronomical telescopes
}

Derek Sabatke, J. Scott Knight, Matthew R. Bolcar

Derek Sabatke, J. Scott Knight, Matthew R. Bolcar, "Polarization ray tracing and polarization aberration compensation in reflective astronomical telescopes," Proc. SPIE 10743, Optical Modeling and Performance Predictions X, 1074307 (17 September 2018); doi: 10.1117/12.2320759

SPIE Event: SPIE Optical Engineering + Applications, 2018, San Diego, California, United States 


\title{
Polarization ray tracing and polarization aberration compensation in reflective, astronomical telescopes
}

\author{
Derek Sabatke ${ }^{\mathrm{a}}$, J. Scott Knight ${ }^{\mathrm{a}}$, and Matthew R. Bolcar ${ }^{\mathrm{b}}$ \\ ${ }^{a}$ Ball Aerospace, 1600 Commerce Street, Boulder CO 80301 USA

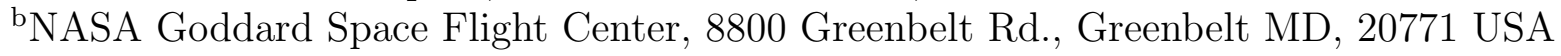

\begin{abstract}
Assessing and optimizing polarization performance in the context of ray-based optical design can be challenging. We describe an approach to this problem that decouples polarization effects from optical system geometry for reflective systems. Each surface's polarization properties are parameterized in terms of their impact on retardance and diattenuation in the small angle-of-incidence limit, separating polarization assessment from the task of coating design. A low-resolution ray trace of the system is adequate to determine ray geometry at each interface, which can then be interpolated to rapidly evaluate net Jones Matrix pupil functions. Coating behavior can be easily varied using the ellipsometric parameters to investigate impacts and compensation. Desired values of these parameters can then be specified as constraints in coating design. Investigation with candidate telescope optical designs for LUVOIR show baseline root-mean-square wavefront errors in the nm range for the on-diagonal Jones matrix component, and throughputs of tens of parts per million. Promising possibilities for compensation using a purpose-designed coating on the secondary mirror are discussed, which reduce the on-diagonal wavefront error by a factor $\sim 20$, with accompanying but more modest reductions in coupling into off-diagonal terms.
\end{abstract}

Keywords: polarization, aberration, compensation, LUVOIR

\section{OVERVIEW}

While the number of known exoplanets has burgeoned in recent years, Earth-like rocky planets tend to be elusive due to their relatively small size, mass and orbital radius. Furthermore, confirmation of a planet's existence is no longer the end goal - it is desirable to characterize exoplanets' composition and atmospheres, which may indicate signs of life. This sort of direct detection is accomplished with a coronagraph, which in currently envisioned systems such as the LUVOIR Surveyor ${ }^{1}$ will need the ability to suppress starlight by a formidable 10 orders of magnitude to adequately characterize the light from an earth-like planet.

Such a level of suppression requires a comprehensive systems architecture. The expected performance is a function of the optical prescription, structural components, expected thermal loading, excitation dynamics, and more. Multiple authors have identified polarization aberrations as one of the challenges to exoplanet direct detection with a coronagraph. ${ }^{2-5}$ Like wavefront aberrations, polarization aberrations arise from the interaction of light with optical surfaces, and have the potential to degrade system performance. In this paper, we treat polarization aberrations of imaging systems in a ray-based context that allow them to be considered alongside conventional wavefront aberrations.

\section{BACKGROUND}

\section{1 pupils and aberrations}

In conventional ray tracing, a bundle of rays is traced from a given field point to an image. Ideally, the rays would converge to a point, and the optical path length along each ray to a sphere centered on that point would be the same for every ray. By choosing a reference sphere and measuring actual optical path length (Fig 1), we determine the wavefront error for each ray. ${ }^{6}$ This can be taken as a function $W(u, v)$ of position in the system's exit pupil coordinates $u, v$.

Further author information:

D.S.: E-mail: dsabatke@ball.com 


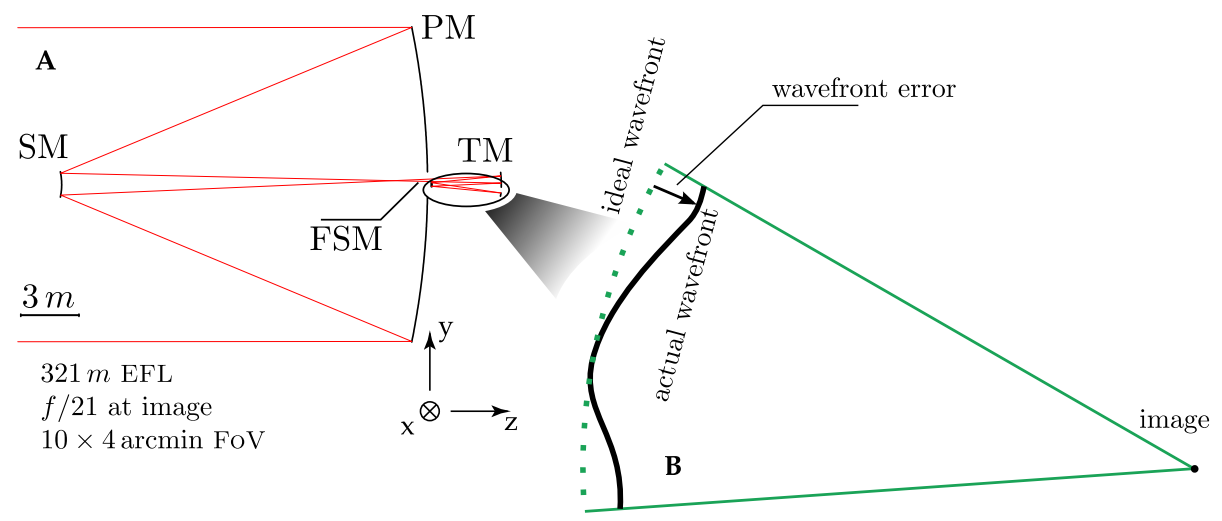

Figure 1. Layout (A) of a $15 \mathrm{~m}$ entrance pupil diameter telescope such as considered in this paper, with basic data and the primary mirror coordinate axes indicated for reference. The zoom image in $\mathbf{B}$, which illustrates the definition of geometrical wavefront error, is not to scale.

Such a geometric result can then be easily married to diffraction analysis. The wavefront error is combined with a pupil amplitude function $A(u, v)$ to give the complex-valued pupil function $P(u, v)=A(u, v) e^{i 2 \pi W(u, v) / \lambda}$. Propagation between exit pupil and image planes amounts to computing a Fourier transform. Taking the absolute magnitude squared of the electric field distribution at the image gives the irradiance point spread function (PSF). ${ }^{7}$

This picture generalizes readily when we include polarization. ${ }^{4,8,9}$ The complex pupil function becomes a $2 \times 2$-element Jones matrix, which we denote

$$
\boldsymbol{P}(u, v)=\left(\begin{array}{rr}
P_{x x}(u, v) & P_{x y}(u, v) \\
P_{y x}(u, v) & P_{y y}(u, v)
\end{array}\right)
$$

Without loss of generality we may factor this as

$$
\boldsymbol{P}(u, v)=A e^{i 2 \pi W / \lambda}\left(\begin{array}{cc}
1 & A_{x y} e^{i 2 \pi W_{x y} / \lambda} \\
A_{y x} e^{i 2 \pi W_{x y} / \lambda} & A_{y y} e^{i 2 \pi W_{y y} / \lambda}
\end{array}\right)
$$

pulling out an overall amplitude and wavefront error to play a role analogous to the conventional versions, and leaving the other terms to show differences from the $x x$ term. Note that the overall wavefront $W$ contains the contributions of both the geometrical wavefront and polarization effects present in the $P_{x x}$ term.

\section{2 polarization due to reflection}

Polarization aberrations have their physical origin in the phase shift and amplitude attenuation that a ray undergoes at each reflection in the system. (Similar effects occur at transmissive interfaces, though we restrict our attention to reflective systems here.) For a broad class of reflectors (including a dielectric material, bulk metal, or planar and isotropic coating), the incoming and outgoing polarization can be related by the Jones vector

$$
\boldsymbol{E}_{\text {out }}=\left(\begin{array}{cc}
A_{p} e^{i \delta_{p}} & 0 \\
0 & A_{s} e^{i \delta_{s}}
\end{array}\right) \boldsymbol{E}_{\text {in }}
$$

where the matrix applies in the $\mathrm{p}, \mathrm{s}$ system, ${ }^{10}$ constructed from the plane of incidence as in Fig $2 \mathbf{A}$.

The absolute normalization of this matrix can be difficult to determine in practice. In ellipsometry (the laboratory characterization of coatings) it is common to factor eq 3 as

$$
\boldsymbol{E}_{\text {out }}=A_{s} e^{i \delta_{s}}\left(\begin{array}{cc}
\tan \Psi e^{i \Delta} & 0 \\
0 & 1
\end{array}\right) \boldsymbol{E}_{\text {in }} .
$$



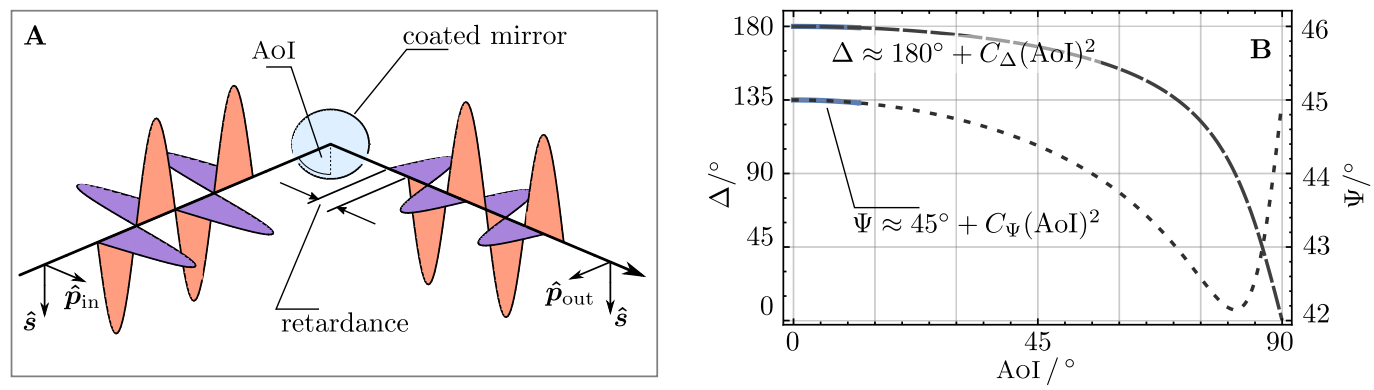

Figure 2. Polarization effects due to reflection from an interface are readily analyzed in the $p, s$-plane (A). Retardance describes the difference in phase delay between eigenpolarizations. Symmetry dictates quadratic behavior at small anglesof-incidence for both ellipsometric angles $\Psi$ and $\Delta$ plotted in $\mathbf{B}$. The curves shown describe a $17 \mathrm{~nm}$ layer of LiF on $\mathrm{Al}$ at a wavelength of $1 \mu \mathrm{m}$.

Here $\Psi$ and $\Delta$ are the ellipsometric angles, defined by

$$
\tan \Psi \equiv\left|A_{p} / A_{s}\right|, \Delta \equiv \delta_{p}-\delta_{s} .
$$

The external factor of $A_{s} e^{i \delta_{s}}$ in Eq 4 can be neglected when overall throughput and phase delay are not under consideration (as in this study).

For a bulk material the parameters $A_{p}, A_{s}, \delta_{p}, \delta_{s}, \Psi, \Delta$ in the foregoing equations are determined by the Fresnel formulae. For coated surfaces they are calculated using well established methods from the complex index of refraction and thickness of each layer of the coating. ${ }^{11}$

We note that at normal incidence, symmetry dictates that $\Psi=(\pi / 4) \mathrm{rad}=45^{\circ}$ (equal amplitudes) and $\Delta=\pi \mathrm{rad}=180^{\circ}$ (no physical phase difference but a flipped coordinate axis). ${ }^{12,13}$ Furthermore, as illustrated in Fig 2B the departure from these values for small angle of incidence (AoI) has no first order term, enabling the approximations

$$
\begin{aligned}
& \Psi \approx \pi / 4+C_{\Psi}(\mathrm{AoI})^{2} \quad \text { for small AoI } \\
& \Delta \approx \pi+C_{\Delta}(\mathrm{AoI})^{2}
\end{aligned}
$$

Here $C_{\Psi}$ and $C_{\Delta}$ are ad hoc coefficients describing the quadratic dependence of $\Psi$ and $\Delta$ on AoI respectively. These coefficients carry units such as inverse radians, and are in general dependent on coating prescription and wavelength. They prove to be a convenient way of parameterizing the polarization-dependent behavior of coatings for our design study.

\section{EVALUATION BY RAY TRACING}

The polarization behavior of an optical system is determined by applying Eq 3 at each reflection. Account must be taken of the geometry (AoI and and plane of incidence) of each ray in the traced bundle at each surface. Ray tracing software generally is able to do this automatically, returning a final result in a common coordinate system at the exit pupil. In our case we use Code V, ${ }^{14}$ looping across the pupil using its rsi ray trace command and mining required results from the ray database via a MAтнематісA ${ }^{15}$ script. $^{2}$

Our particular interest at present lies with candidate telescope designs for LUVOIR. A number of optical designs of Cassegrain and three mirror anastigmat (TMA) design forms have been developed and evaluated in the course of that team's efforts. We focus our presentation on two of the more mature candidates: an obscured system with $15 \mathrm{~m}$ entrance pupil diameter (EPD) shown in Fig 1A and an unobscured $8 \mathrm{~m}$ design (introduced in Fig 6). The primary mirror (PM) will be segmented, although the segmentation is not relevant to our work so we use a monolithic optical surface for simplicity. These telescopes were optimized subject to constraints restricting AoI to no more than $15^{\circ}$. This limit was reached for the PM and secondary mirror (SM), while the tertiary and fine steering mirrors (TM and FSM) have only small AoIs. Thus the PM and SM likely drive these systems' polarization aberrations. 

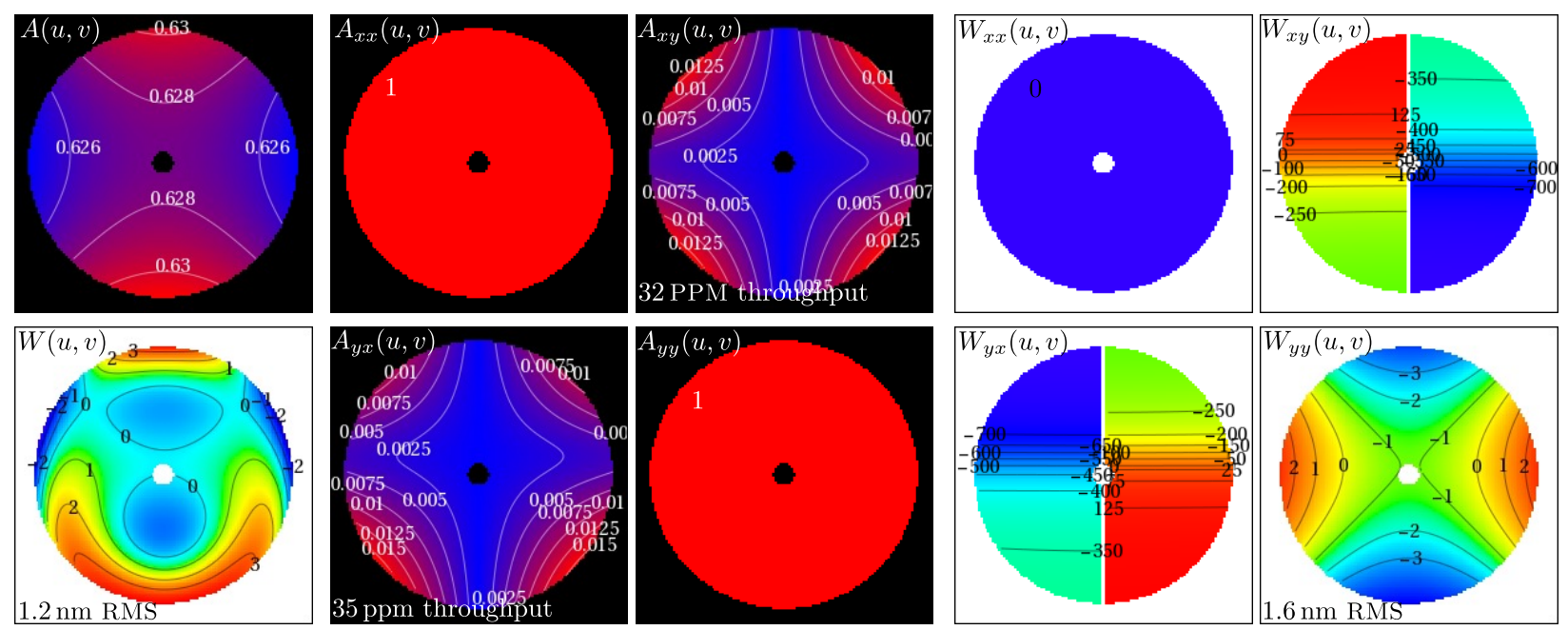

Figure 3. An example set of Jones pupil functions, evaluated for a $15 \mathrm{~m}$ optical design, for a field location with 0, -1.35 millirad line-of-sight in PM coordinates. The layout is meant to be evocative of factoring in eq 2 , with amplitude terms on a black background and wavefronts on a white background. Phasemap contours are labeled in nm units.

Fig 3 shows a representative Jones pupil (from the $15 \mathrm{~m}$ design) at an on- $y$-axis field point. Contour plots in the figure are laid out to evoke the factoring of Eq 2. Amplitude terms are shown on a black background with a blue-to-red color scale; phase maps are shown on a white background with a rainbow color scale. The $A_{x x}$ and $W_{x x}$ terms are ideal by virtue of factoring. The $A_{y y}$ amplitude term is nearly ideal as well. Variations in the overall and on-diagonal amplitude terms are small and appear to be less of a concern than phase effects and off-diagonal amplitudes. The system will include a deformable mirror for adaptive correction of wavefront errors in at least one coronagraph channel. Hence we can understand the overall phase factor in Eq 2 to have been nulled, and focus our attention on the contents of the matrix.

The $W_{y y}$ phasemap shows prominent astigmatism aligned to the major axes. At $1.4 \mathrm{~nm}$ RMS, the magnitude of the WFE is small by conventional standards, but nonetheless potentially concerning for coronagraphy. The off-diagonal wavefronts are complex, but are gated by amplitude terms with very small values. The amplitudes tend to be greatest near the edges of the pupil, where the corresponding wavefronts have plateaus at different extreme values, creating a stair step effect.

Assessing the significance of the off-diagonal pupil terms is a difficult subject, which deserves and receives considerable separate attention. ${ }^{5,9}$ Incorporation of these results in a coronagraph physical optics model to assess final coronagraph performance is advisable (and in our case is undertaken by other members of the LUVOIR team). Nevertheless, it's worth keeping a few top-level considerations in mind to inform our intuition.

Each Jones matrix pupil term contributes a corresponding point spread function. For a first assessment, we may consider these to add incoherently. ${ }^{3}$ Summing down a column of the Jones matrix is valid in computing a final irradiance image, since the two components represent orthogonal polarizations which do not interfere (though of course the telescope prime image is not the final image). Summing along a row amounts assuming that input electric field components are incoherent, that is that randomly polarized light is input. Although polarimetry is an important technique in exoplanet detection, low degrees of polarization are expected and such an assumption is the most reasonable one to be made without a detailed source model. The PSFs are influenced by pupil amplitude and wavefront variations in the same manner as in the scalar case. Thus our intuitions about wave propagation and image formation are expected to translate term-by-term from the scalar case.

Where they can be applied, image quality metrics such as RMS WFE carry their significance over to the polarized case. Instances arise however (such as the second field shown in fig 5 ) where it is difficult to identify a well-behaved wavefront function. There the off-diagonal wavefronts exhibit a $\pi$ radian discontinuity. This does not indicate a discontinuity in the physical behavior of the system. The wavefront discontinuity occurs at a 

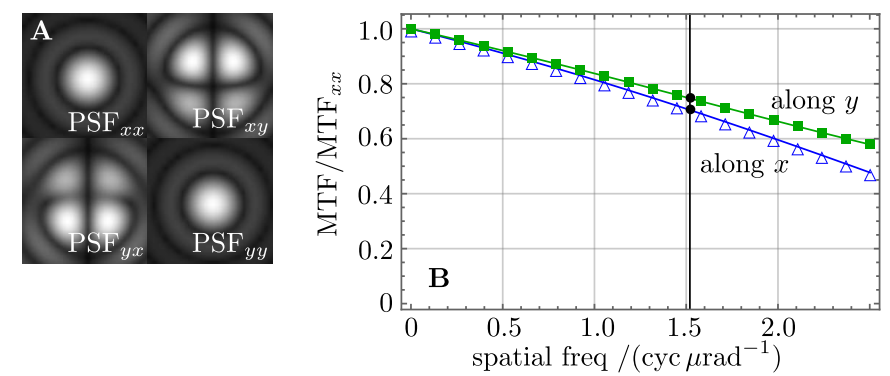

Figure 4. Point spread functions (A) obtained by Fourier analysis of the corresponding Jones pupil maps in Fig 3 . Images are normalized in intensity and adjusted in contrast to make detail visible. Note that the off-diagonal elements have substantially lower throughput than their on-diagonal counterparts. Part B shows profiles of the $x y$ term's PSF, with those of the $x x$ term normalized out to remove the nominal impact of diffraction due to the system aperture. The vertical line indicates a nominal reference spatial frequency corresponding to $D /(10 \lambda)$.

position in the pupil where the amplitude is zero, so for example the combined complex functions $A_{x y} e^{i 2 \pi W_{x y} / \lambda}$ is continuous. It's possible to eliminate the discontinuity by allowing a negative-going amplitude, leaving a smooth behavior in both $A_{x y}$ and $W_{x y}$, with a smaller RMS WFE. However this simply means there is a phase effect not encapsulated by the wavefront. Since the off-diagonal terms tend to have large wavefront errors, their overall throughput is likely to be very important.

Fig 4A shows the PSFs obtained via Fourier analysis of the maps in Fig 3, with throughput normalized and contrast adjusted to make the tails visible, and corresponding modulation transfer function (MTF) profiles. Finding a simple numerical figure-of-merit to describe the off-diagonal terms' impact on image quality is not easy. We report RMS WFE in some cases, however it is not necessarily meaningful where wavefronts have $\pi$ rad phase discontinuities or amplitudes have been allowed to run negative. As an added complication, the off-diagonal pupil amplitudes tend to have small values in near center, so we apply the square of the amplitude as a weight factor in computing RMS WFE for these cases.

Other common image quality metrics are likewise poorly suited to the task of describing the off-diagonal spots. RMS spot radius diverges for diffraction-driven PSFs. Strehl ratio ${ }^{16}$ (where we use the ideal spot of an open pupil such as the $J_{x x}$ term for normalization) tends to be zero for these PSFs since they show destructive interference on center. The most promising metric to present itself is one based on MTF. As shown in Fig 4, we normalize out the $J_{x x}$ term's MTF to remove diffraction effects due to the system aperture. Therefore we expect constant traces at unity for ideal performance, and a quadratic roll off for small aberrations. The fact that normalized MTF plots have a linear trend at the origin indicates that these terms in effect apply a binary phase mask which reduces the pupil diameter driving diffraction - an effect that is clearly observable in the PSFs as well. As an aid to comparing results from the two systems of differing field points and aperture diameter, the black vertical line in the MTF plots shows the spatial frequency $D /(10 \lambda)$ - one tenth the cut-off spatial frequency for a diffraction-limited, incoherent system.

Figs 5 and 6 expand the library of Jones pupil maps with, respectively, additional field points in the $15 \mathrm{~m}$ model and a representative result from the $8 \mathrm{~m}$ system. There is no fundamental constraint on these Jones matrices that they display Hermitian symmetry, and careful inspection of the plots in this paper show symmetry violations. However most of our results are at least qualitatively symmetric, so to save space and more clearly emphasize relevance in the results, we omit some maps from the figures we present. It is worth noting that even for off-axis field points, the $J_{y y}$ astigmatism tends to be constant with field in the $15 \mathrm{~m}$ case. In the $8 \mathrm{~m}$ case, the $J_{y y}$ displays astigmatism, but tilt dominates. Although the off-diagonal amplitude terms vary considerably, there are sign flips evident in at least one axis in all cases.

\section{PARAMETRIC INTERPOLATION}

The polarization aberration results of the previous section were evaluated via ray tracing. This requires explicit choices of wavelength and coating design or designs in advance, and is unwieldy for conceptual exploration. We 


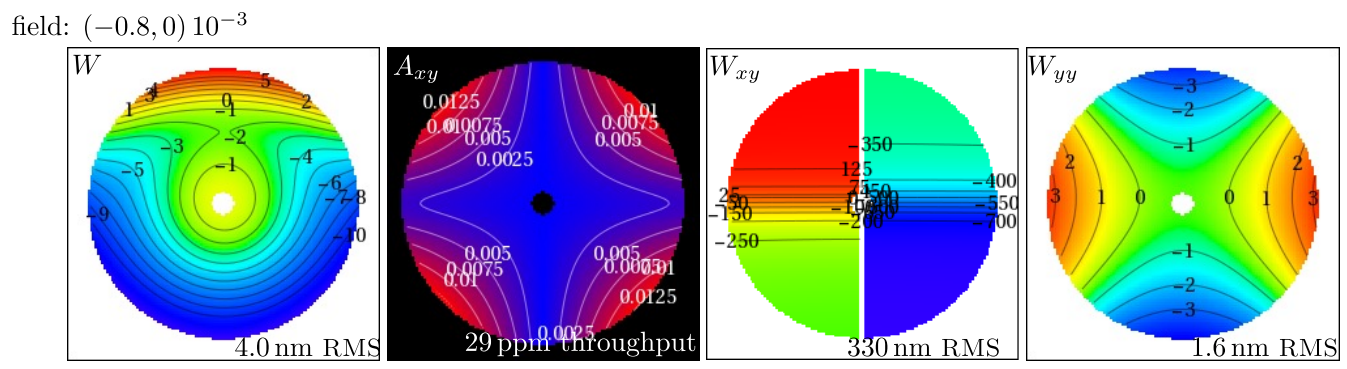

field: $(-1.2,1.2) 10^{-3}$
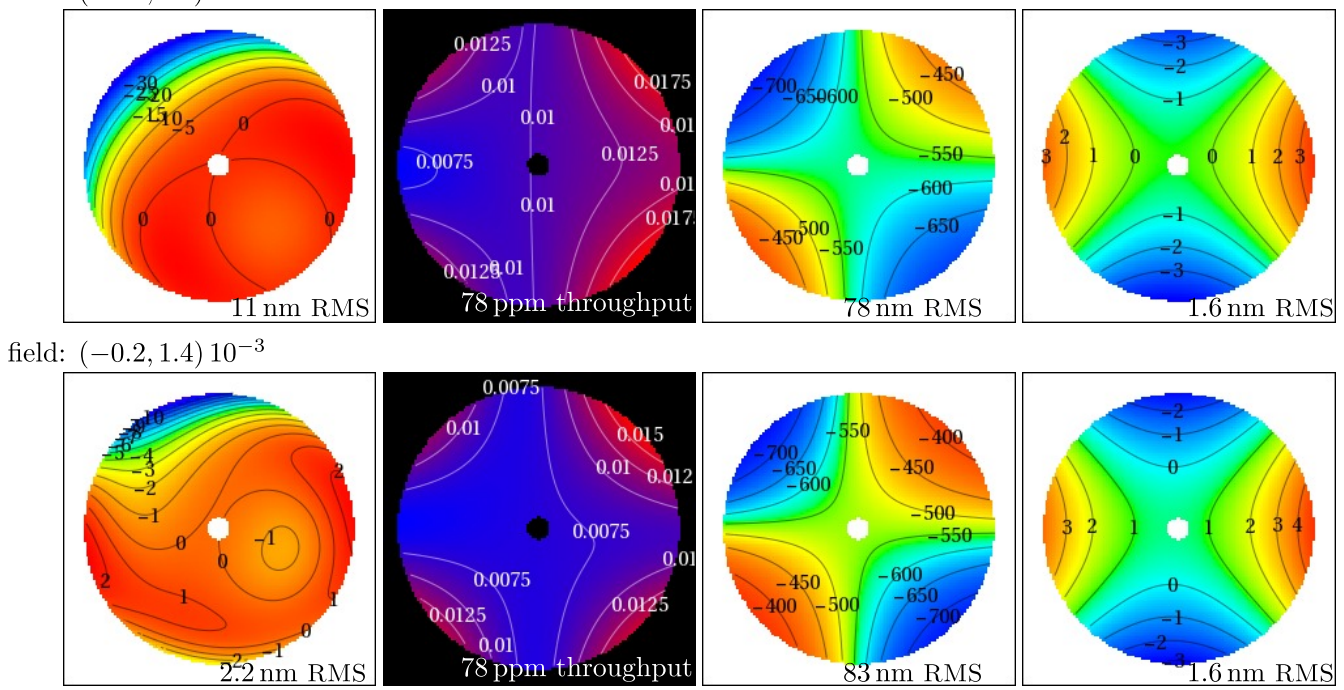

Figure 5. A selection of additional results for the $15 \mathrm{~m}$ system shown in Fig 3. All mirrors have $17 \mathrm{~nm}$ of LiF on Aluminum coatings, and wavelength is set to $1 \mu \mathrm{m}$. Field points are given as outward looking direction cosines relative to the axes of the PM parent surface, shown in Fig 1. Phase map contours are labeled in units of nm. Some maps are omitted for brevity.
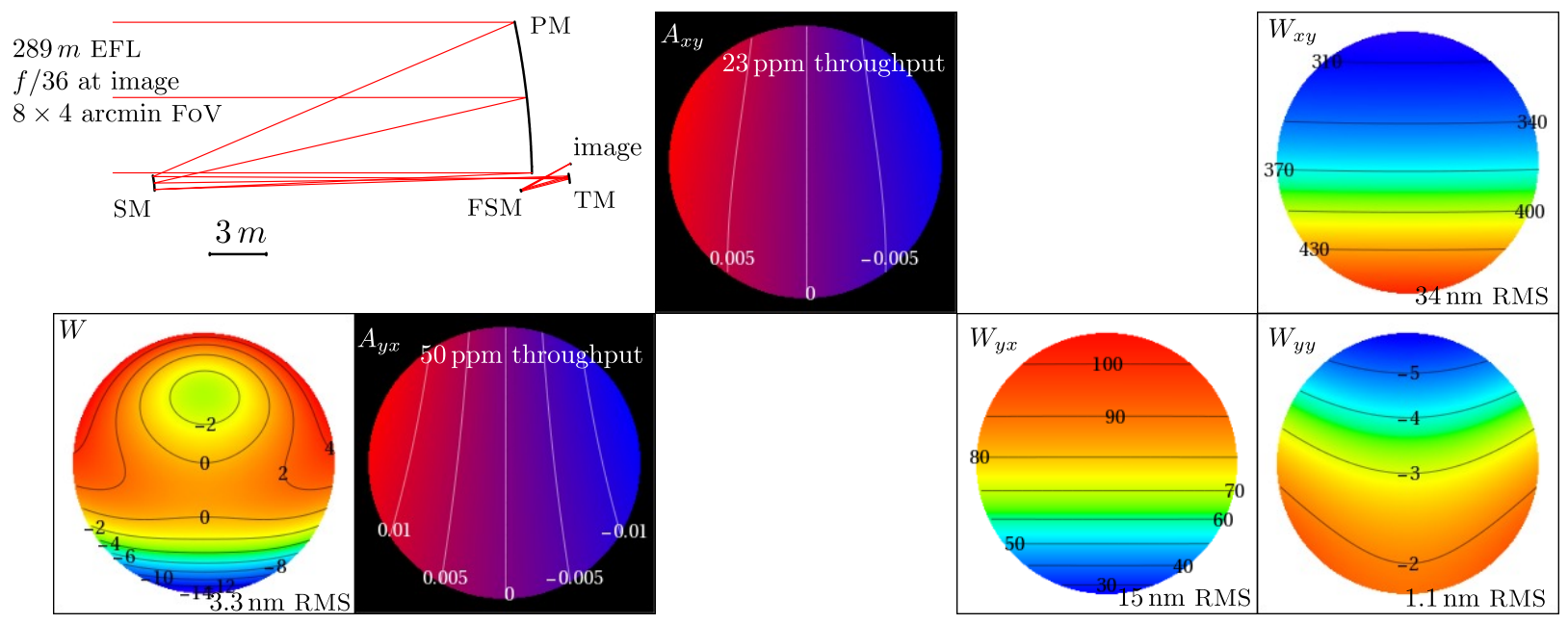

Figure 6. Telescope layout and system performance for the $8 \mathrm{~m}$ EPD design. Results are shown for the coronagraphy field point (having line-of-sight direction cosines of $\left(0,-1.5 \times 10^{-3}\right)$ ) at a wavelength of $1 \mu \mathrm{m}$. 
found it helpful to build a model which was a hybrid of Jones matrix mechanics carried out in Mathematica and interpolated ray tracing and coating results from Code V. Because the the system is reflective and the polarization aberrations under consideration are small, to an excellent approximation ray paths do not depend on wavelength or coating selection. Moreover, for the systems under consideration, ray geometry is slowly and smoothly varying with pupil and field, so a modest number of traced rays is sufficient to characterize it.

For each evaluated point in the pupil, the net Jones matrix is computed by simple matrix product, with one factor for each of $N$ reflections (with $N=4$ for most cases in our study).

$$
\boldsymbol{J}_{\text {net }}=\ldots \boldsymbol{J}_{4} \boldsymbol{J}_{3} \boldsymbol{J}_{2} \boldsymbol{J}_{1}=\prod_{i=0}^{N-1} \boldsymbol{J}_{N-i}
$$

The factor of interest is the Jones matrix given in Eq 4 with an appropriate coordinate system transformation:

$$
\boldsymbol{J}_{k}=\left(\begin{array}{ll}
\boldsymbol{x}_{k} & \boldsymbol{y}_{k}
\end{array}\right)\left[A_{s, k} e^{i \delta_{s, k}}\left(\begin{array}{cc}
\tan \Psi_{k} e^{i \Delta_{k}} & 0 \\
0 & 1
\end{array}\right)\right]\left(\begin{array}{c}
\boldsymbol{p}_{\mathrm{in}, k}^{t} \\
\boldsymbol{s}_{k}^{t}
\end{array}\right)
$$

Here the notation $\boldsymbol{v}^{t}$ represents the row vector obtained by transposing a column vector $\boldsymbol{v}$. The notation on the right means to expand the vectors $\boldsymbol{p}_{\mathrm{in}, k}^{t}$ and $\boldsymbol{s}_{k}^{t}$ as rows to form a matrix. Similarly the notation on the left means to expand $\boldsymbol{x}_{k}$ and $\boldsymbol{y}_{k}$ as columns.

The vectors $\boldsymbol{p}_{\mathrm{in}, k}$ and $\boldsymbol{s}_{k}$ give the p,s axes for the $k^{\text {th }}$ surface expressed in an exit coordinate system chosen with the $(k-1)^{\text {th }}$ surface. The vectors $\boldsymbol{x}_{k}$ and $\boldsymbol{y}_{k}$ define this exit coordinate system for the $k^{\text {th }}$ surface, expressed in $\boldsymbol{p}_{\text {in }, k}$ and $\boldsymbol{s}_{k}$ coordinates. The exit system may be $\boldsymbol{p}, \boldsymbol{s}_{\text {out }}$ for that surface, or another working system chosen for convenience.

The transformation in Eq 8 is analogous to the familiar Jones matrix rotation relation,

$$
\boldsymbol{J}(\theta)=\left(\begin{array}{cc}
\cos \theta & -\sin \theta \\
\sin \theta & \cos \theta
\end{array}\right) \boldsymbol{J}(0)\left(\begin{array}{cc}
\cos \theta & \sin \theta \\
-\sin \theta & \cos \theta
\end{array}\right) .
$$

This is commonly used with non-deviating, transmissive systems, in winch the only degree of freedom is the element's azimuthal orientation $\theta$. A known, un-rotated Jones matrix $\boldsymbol{J}(0)$ is sandwiched between coordinate transforms to give the Jones matrix $\boldsymbol{J}(\theta)$ describing the component as rotated. However Eq 8 allows the use of three-dimensional coordinate vectors, allowing application to reflections with compound angles-of-incidence and non-collimated ray fans.

To make use of these relations, we begin by tracing a coarse fan of rays in CODE V to determine ray geometry. Since that geometry varies slowly and smoothly, we can interpolate with pupil position to fill in the fan as desired. (Interpolating with field should be similarly possible, though we have not yet implemented it.) To investigate the impact of coating performance, we can select values of $C_{\Psi}$ and $C_{\Delta}$ of interest for each surface. Alternately, to evaluate performance with particular coatings, we can have CODE $\mathrm{V}$ evaluate coating prescriptions for a range of angles of incidence, fit or interpolate to allow variable AoI, and interpolate with wavelength. Net system Jones pupil functions are then obtained by composing the foregoing relations and evaluating the Jones matrix product. This gives us a way of cross-checking and probing the end-to-end polarization calculations carried out by CODE V. Conversely, our math is readily checked by carrying out a full trace there for a case with an in-hand coating prescription. With our parametric model we can rapidly explore drivers and options for compensation, even wrapping frequently varied parameters such as $C_{\Psi}$ and $C_{\Delta}$ or wavelength with graphical sliders for interactive operation.

\section{COMPENSATION VIA COATINGS}

Fig 7A gives a first demonstration of the parametric model, in the form of a complete sweep of performance against wavelength for the $8 \mathrm{~m}$ design. Part $\mathbf{B}$ displays selected maps in full at $\lambda=1 \mu \mathrm{m}$. These maps may be compared directly to the ray trace results in Fig 6 as a validation of the model, and show excellent agreement. 

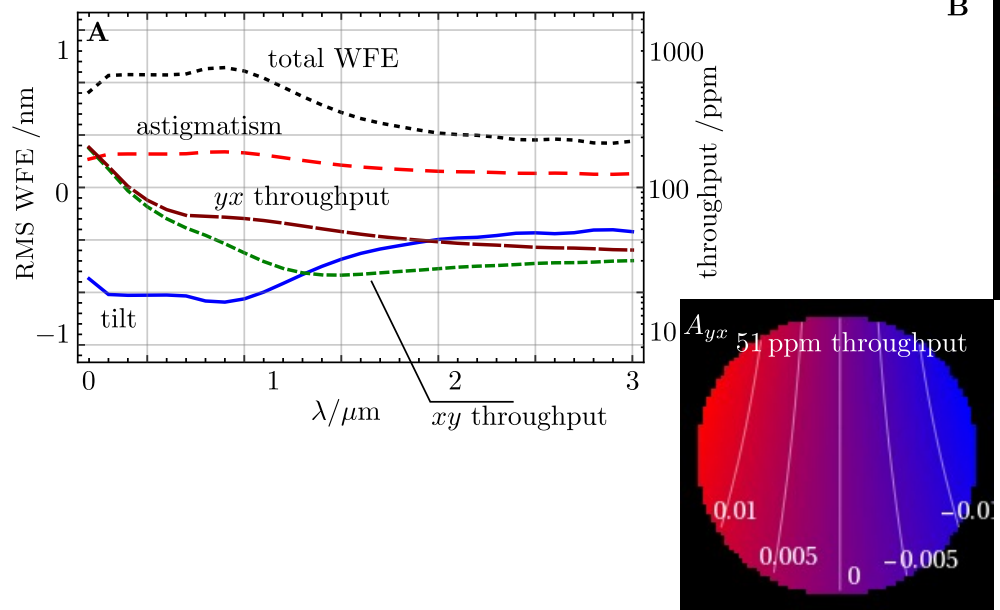
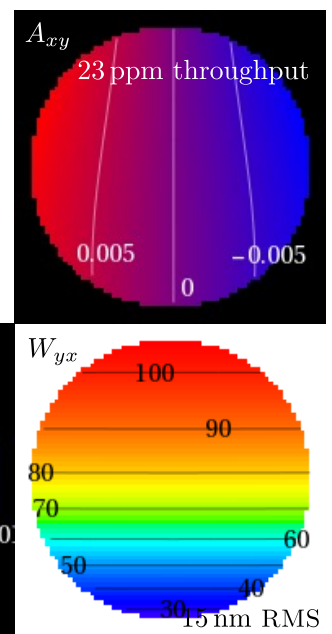
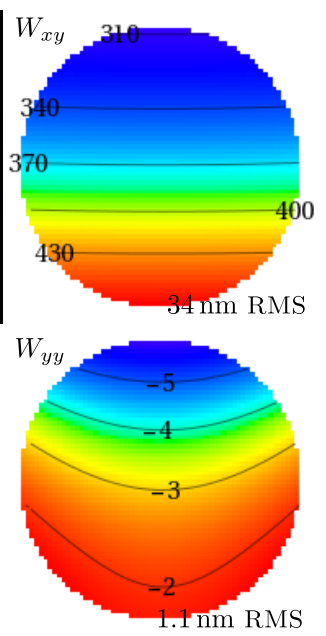

Figure 7. Wavelength trending of RMS WFE and off-diagonal throughput for the $8 \mathrm{~m}$ design, generated with the parametric model (A). Part B shows selected Jones pupil maps for $\lambda=1 \mu \mathrm{m}$, which exhibit close agreement with the ray-tracing results of Fig 6 .
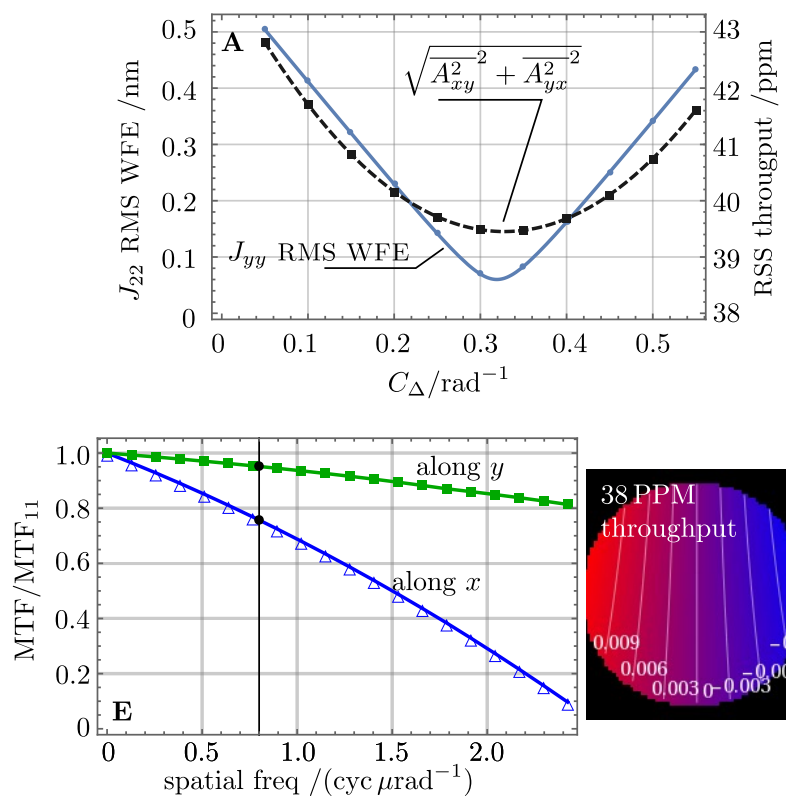

B $\quad 11$ PPM throughput
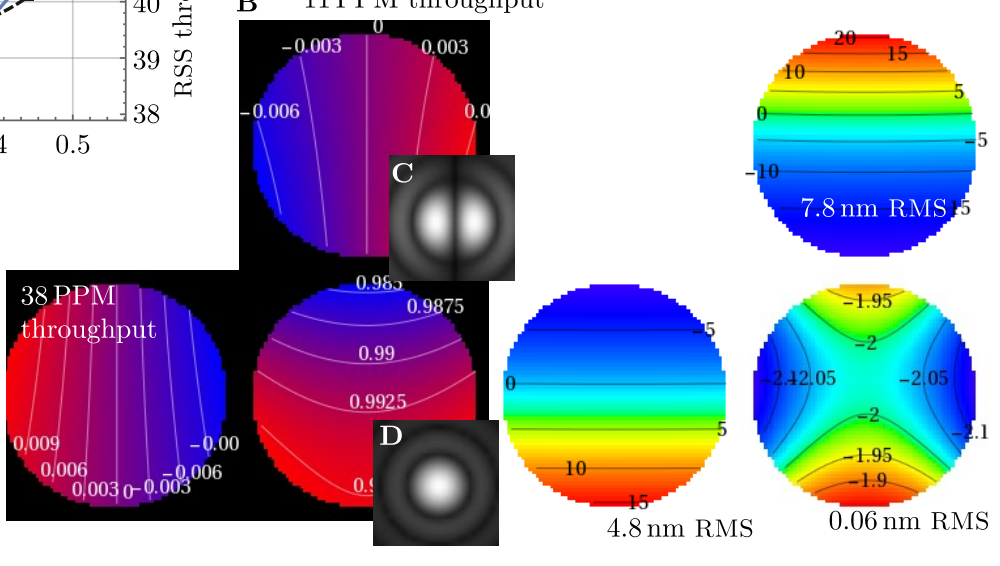

Figure 8. Varying the retardance coefficient $C_{\Delta}$ for the secondary mirror of the $8 \mathrm{~m}$ design (at the coronagraph field point and $1 \mu \mathrm{m}$ wavelength) reveals a minimum in the $J_{y y}$ wavefront error $(\mathbf{A})$. The remaining sub-figures indicate system performance at the optimum $C_{\Delta} \approx 0.32 \mathrm{rad}^{-1}$. These include Jones pupil maps (B), selected Jones term PSFs (C and $\mathbf{D})$, and relative MTF profiles for the $J_{y x}$ term $(\mathbf{E})$. Power-law mapping has been applied to the spot images in $\mathbf{C}$ and $\mathbf{D}$ to make the Airy rings visible.

Parameterizing coating behavior gives a practical way of exploring aberration compensation. Fig $8 \mathbf{A}$ shows a plot of $J_{y y}$ wavefront error as $C_{\Delta}$ is varied on the secondary mirror, using a $1 \mu \mathrm{m}$ wavelength and $17 \mathrm{~nm}$ of $\mathrm{LiF}$ on $\mathrm{Al}$ on the other mirrors. The remainder of the figure is a montage of performance metrics evaluated at the optimum $\left(C_{\Delta} \approx 0.32 \mathrm{rad}^{-1}\right)$. Note that the $J_{y y}$ WFE is considerably improved over the baseline performance shown in Figs 6 and 7, having its RMS variation reduced by a factor of 18, largely eliminating the dominant tilt term and reducing the astigmatism. 


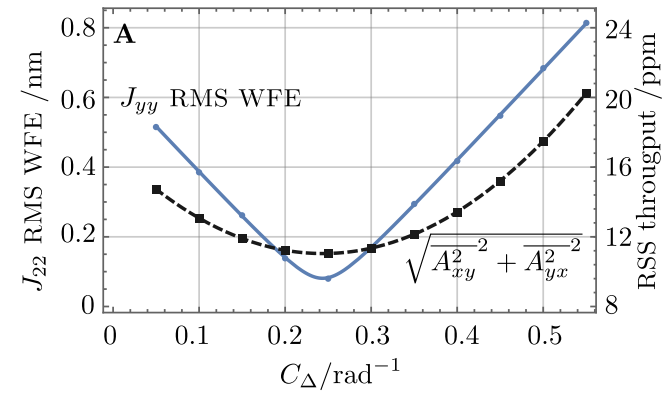

\section{B $\quad 6.2 \mathrm{ppm}$ throughput}
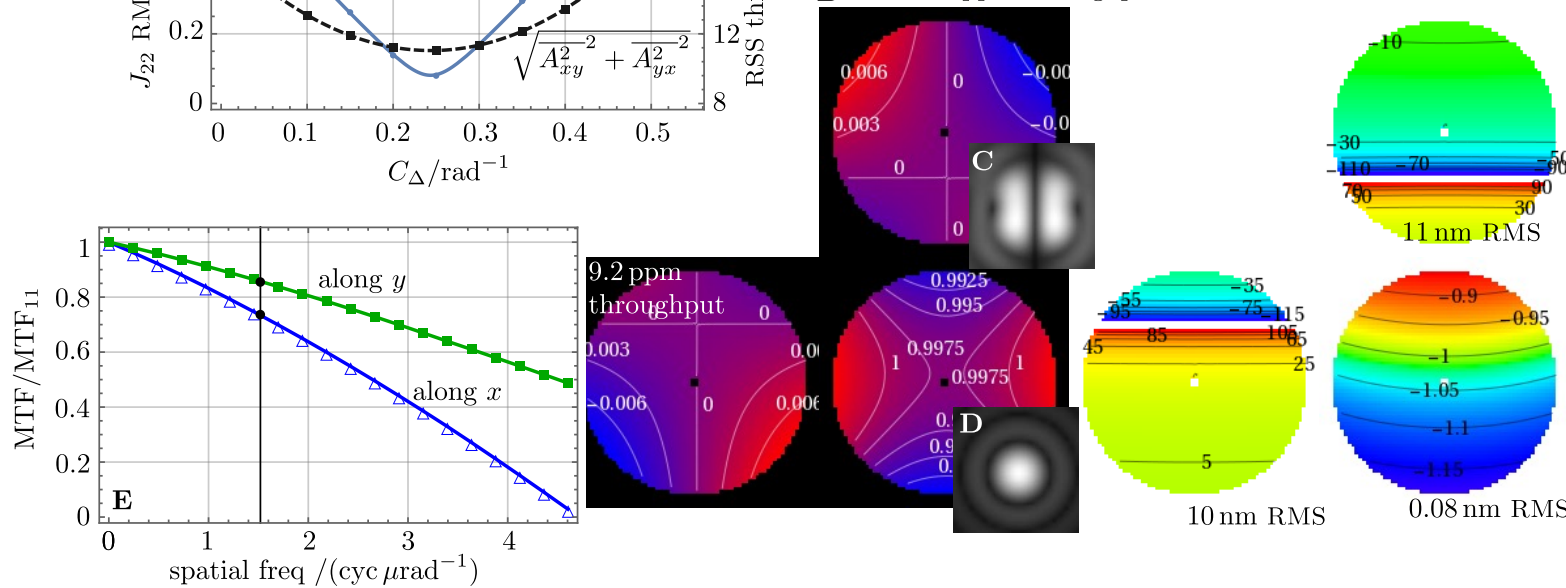

$10 \mathrm{~nm}$ RMS

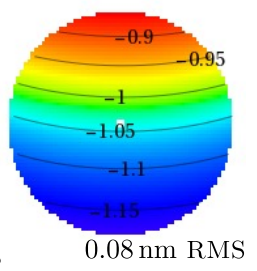

Figure 9. Retardance optimization results for a $15 \mathrm{~m}$ model (A). Performance results in $\mathbf{B}-\mathbf{E}$ at the location of minimum $J_{y y}$ RMS WFE at $C_{\Delta} \approx 0.24 \mathrm{rad}^{-1}$ are presented in the same manner as the $8 \mathrm{~m}$ case of Fig 8 .

The RMS WFE of the off-diagonal terms is also improved. The PSFs for the $x y$ and $y x$ terms are not substantially improved in their shape. This is evidenced by the PSF in $\mathbf{C}$ (which can be compared to the diffractionlimited spot in D) and the normalized MTF profiles in E. (The two cases have practically indistinguishable spot shapes, so only selected MTF and PSF plots are shown.) Perhaps the most important consideration for the off-diagonal terms is throughput, and we note that they are both improved. In fact, the dashed curve in fig $8 \mathbf{A}$ shows the RSS throughput of these terms, revealing that the $J_{y y}$ optimum is very close to simultaneously optimizing (that is, minimizing) off-diagonal throughput.

Fig 9 presents comparable results for the $15 \mathrm{~m}$ model. It similarly shows improvement in $J_{y y}$ RMS WFE and off-diagonal throughput, though with a somewhat different location of the optimum. The residual wavefront errors are slightly larger, while the off-diagonal throughput values are smaller. These results are perhaps not surprising in light of the larger aperture and optical design which is closer to being on axis.

We chose the SM to carry the compensation for these exercises, since for the moment we have only a telescope model available to work with. We do not mean to suggest such an option for the actual system design, since tampering with a telescope coating impacts throughput and polarization aberrations for all instruments and wavelengths. In particular, overcoat thickness on these mirrors is an important consideration for UV throughput. A far preferable approach would be to apply compensation on an internal optic of each science instrument as required. Studying the feasibility of such an approach remains for further work, when instrument and coronagraph models are available.

It is nonetheless interesting to evaluate the performance of the compensation over a range of wavelengths around the $\lambda=1 \mu \mathrm{m}$ design value. Doing so meaningfully requires a coating design rather than a single $C_{\Delta}$ value. For expedience, we limit ourselves at present to varying the overcoat thickness of our $\mathrm{LiF}$ on $\mathrm{Al}$ case. Fig 10A shows how $C_{\Delta}$ and $d C_{\Delta} / d \lambda$ vary with coating thickness at the nominal wavelength, indicating that $209 \mathrm{~nm}$ is the smallest topcoat thickness providing the desired $C_{\Delta}$. Serendipitously, this design also has negative $d C_{\Delta} / d \lambda$, suggesting a trend toward wavelength compensation. Fig 10B shows performance trending with wavelength for such a system ( $209 \mathrm{~nm}$ of $\mathrm{LiF}$ on $\mathrm{Al}$ on the secondary, and $17 \mathrm{~nm}$ on the other mirrors). The compensation is admirably broad, with an effective a range of approximately $0.7-1.1 \mu \mathrm{m}$ or a $\Delta \lambda / \lambda$ of $40 \%$. 

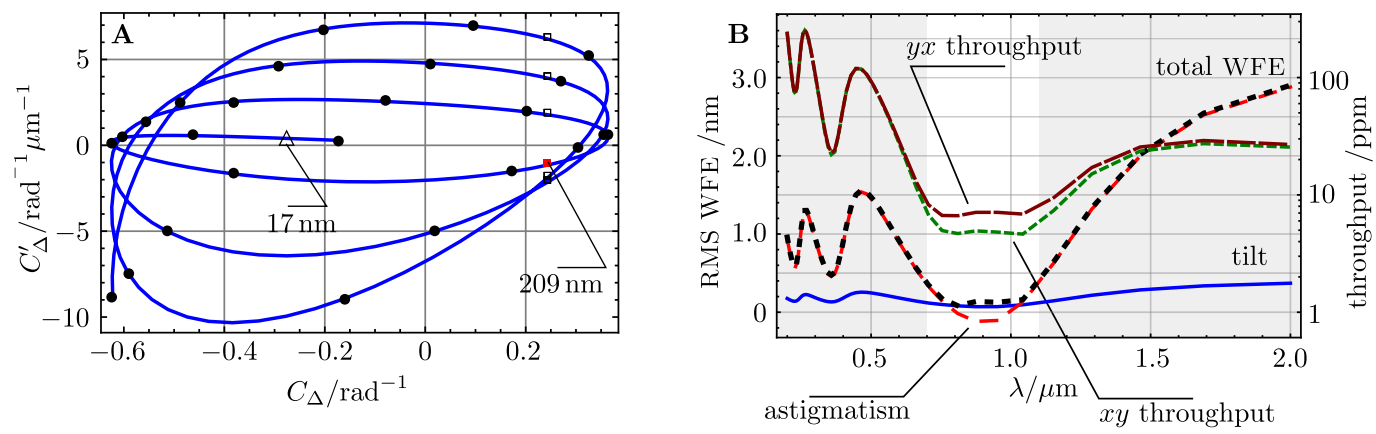

Figure 10. A: Coating performance for LiF-overcoated $\mathrm{Al}$ at $\lambda=1 \mu \mathrm{m}$, plotted in $C_{\Delta}, d C_{\Delta} / d \lambda$ space as a function of coating thickness. Black dots indicate $50 \mathrm{~nm}$ intervals. A top coat thickness of $209 \mathrm{~nm}$ achieves the value $C_{\Delta} \approx 0.24 \mathrm{rad}^{-1}$ selected in Fig 9. B: Performance trending across wavelength for such a system, with $209 \mathrm{~nm}$ of LiF on $\mathrm{Al}$ on the secondary, and $17 \mathrm{~nm}$ on the other mirrors.
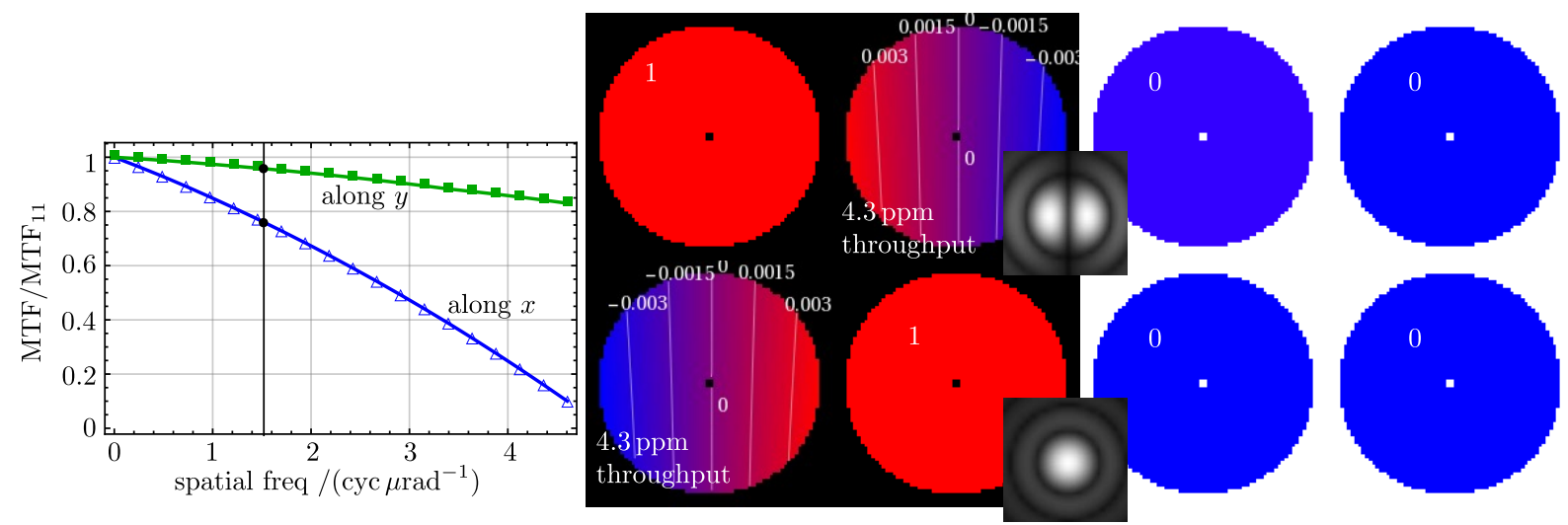

Figure 11. Results for the $15 \mathrm{~m}$ model with ideal coatings $\left(C_{\Delta}=C_{\Psi}=0\right)$ applied. Note that the off-diagonal amplitude elements are not exactly zero, but show a residual of the design's ray geometry which introduces a polarization rotation that varies approximately linearly across the pupil between \pm 4 millirad extremes.

As a final demonstration of the utility of the parametric model, consider the system performance with perfect coatings. Setting $C_{\Delta}=C_{\Psi}=0$ generates the results shown in Fig 11. These show unit amplitude for the on-diagonal throughputs and zero wavefront errors, as may be expected. Note however that the off-diagonal amplitudes are not zero. They show an approximately linear trend which may be identified as a polarization rotation, varying between extremes of about \pm 4 millirad. This residual is presumably a feature of the optical design, and results in about $4 \mathrm{ppm}$ of off-diagonal throughput. This may help to explain the floor reached by off-diagonal throughput in the optimization sweeps of figures $8 \mathbf{A}$ and $9 \mathbf{A}$.

\section{CONCLUSIONS}

Parametric modeling provides an effective way to include polarization effects in ray-based optical design and modeling. The ellipsometric angles $\Psi$ and $\Delta$ describing diattenuation and retardance respectively provide effective parameters. For small angles-of-incidence such as may be obtained in optical telescopes for astronomy, their dependence on AoI is captured with a single parameter each. With this approach, geometric optical design and coating design can both initially be decoupled from polarization problems. Furthermore, polarization drivers are parameterized in a manner that provides effective compensators and advises coating design, without requiring detailed coating designs in advance. 
In our study of optical designs for LUVOIR, coating-induced retardance on the secondary mirror proved effective as a compensator to reduce the wavefront error for the $J_{y y}$ Jones pupil term. Although it has little impact on the image quality of off-diagonal terms, it is effective in reducing the amplitude of those terms. Applying such compensation in the common path is likely not practicable for an extremely broadband system like LUVOIR. However it may be applicable to other systems, as even a simple coating was effective over a bandwidth having $\Delta \lambda / \lambda \sim 0.4$. In the case of LUVOIR, this work provides a promising indication of the potential for compensation in dedicated science instrument channels with more extensive coating optimization, as mature models for the science instruments and coronagraph become available.

\section{ACKNOWLEDGMENTS}

The authors are indebted to the LUVOIR Science and Technology Definition Team for support and suggestions. This work was supported in part by NASA contract NNG15499015R.

\section{REFERENCES}

[1] LUVOIR Study Team, "The LUVOIR interim report," (2018). NASA technical report https://asd.gsfc.nasa.gov/luvoir/resources/docs/LUVOIR_Interim_Report_Final.pdf.

[2] Balasubramanian, K., Shaklan, S., Give'on, A., Cady, E., and Marchen, L., "Deep UV to NIR space telescopes and exoplanet coronagraphs: a trade study on throughput, polarization, mirror coating options and requirements," in [Society of Photo-Optical Instrumentation Engineers (SPIE) Conference Series], Society of Photo-Optical Instrumentation Engineers (SPIE) Conference Series 8151, 1 (Oct. 2011).

[3] Bolcar, M. R., "Impacts of optical coatings on polarization and coronagraphy," NASA document GSFC-EDAA-TN34382 (2016).

[4] Breckinridge, J. B., "Optics Technologies for LUVOIR \& HabEx: Polarization \& Mirror Count," in [American Astronomical Society Meeting Abstracts], American Astronomical Society Meeting Abstracts 229, 206.02 (Jan. 2017).

[5] J. B. Breckinridge and R. A. Chipman, "Threshold raw retrieved contrast in coronagraphs is limited by internal polarization," JPL document D-100127 (2017).

[6] Sasián, J., [Introduction to Aberrations in Optical Imaging Systems], Cambridge University Press (2013).

[7] Goodman, J., [Introduction to Fourier Optics], McGraw-Hill physical and quantum electronics series, W. H. Freeman (2005).

[8] Chipman, R. A., Lam, W. S. T., and Breckinridge, J., "Polarization aberration in astronomical telescopes," Proc. SPIE 9613, 96130H-96130H-11 (2015).

[9] Breckinridge, J. B. and Chipman, R. A., "Telescope polarization and image quality: Lyot coronagraph performance," in [Society of Photo-Optical Instrumentation Engineers (SPIE) Conference Series], Proc. SPIE 9904, 99041C (July 2016).

[10] Azzam, R. M. A. and Bashara, N. M., [Ellipsometry and Polarized Light], Elsevier, Amsterdam (1987).

[11] MacLeod, H., [Thin-Film Optical Filters, Fourth Edition], Series in Optics and Optoelectronics, CRC Press (2010).

[12] Sabatke, D., von Handorf, R., and Sullivan, J., "Polarization and fold mirrors in application of the Leica Absolute Distance Meter," in [Society of Photo-Optical Instrumentation Engineers (SPIE) Conference Series], Society of Photo-Optical Instrumentation Engineers (SPIE) Conference Series 7461 (Aug. 2009).

[13] Breckinridge, J. B., Lam, W. S. T., and Chipman, R. A., "Polarization Aberrations in Astronomical Telescopes: The Point Spread Function," Proc. Astr. Soc. Pacific 127, 445-468 (May 2015).

[14] Synopsys, Inc., Pasadena, CA, Code V ray tracing software. https://www.synopsys.com/opticalsolutions/codev.html.

[15] Wolfram Research, Inc., Champaign, Ill., Mathematica computational environment. https://www.wolfram.com/.

[16] J. C. Wyant and K. Creath, [Applied Optics and Optical Engineering], vol. XI, ch. "Basic Wavefront Aberration Theory for Optical Metrology", Academic, New York (1992). 